\title{
Power Transformer Incipient Faults Diagnosis Based on Dissolved Gas Analysis
}

\author{
Osama E. Gouda ${ }^{*^{1}}$, Saber M. Saleh ${ }^{2}$, Salah Hamdy EL-Hoshy ${ }^{3}$ \\ ${ }^{1}$ Department of Electrical Power and Machines, Faculty of Engineering, Cairo University \\ ${ }^{2}$ Department of Electrical Power and Machines, Faculty of Engineering, Fayoum University \\ ${ }^{3}$ Middle Egypt electricity zone, Egyptian Electricity Transmission Company, El-Fayoum \\ *Corresponding author, e-mail: Prof_ossama11@yahoo.com
}

\begin{abstract}
Incipient fault diagnosis of a power transformer is greatly influenced by the condition assessment of its insulation system oil and/or paper insulation. Dissolved gas-in-oil analysis (DGA) is one of the most powerfull techniques for the detection of incipient fault condition within oil-immersed transformers. The transformer data has been analyzed using key gases, Doernenburg, Roger, IEC and Duval triangle techniques. This paper introduce a MATLAB program to help in unification DGA interpretation techniques to investigate the accuracy of these techniques in interpreting the transformer condition and to provide the best suggestion for the type of the fault within the transformer based on fault percentage. It proposes a proper maintenance action based on DGA results which is useful for planning an appropriate maintenance strategy to keep the power transformer in acceptable condition. The evaluation is carried out on DGA data obtained from 352 oil samples has been summarized into 46 samples that have been collected from a 38 different transformers of different rating and different life span.
\end{abstract}

Keywords: dissolved gas analysis (DGA), fault diagnosis, power transformer, insulating oil, condition assessment

Copyright $@ 2015$ Institute of Advanced Engineering and Science. All rights reserved.

\section{Introduction}

Power transformers are the most expensive and important equipment in electric power systems that serves to convert the power with different level of voltage. During its lifetime, transformer insulation is exposed to mainly electrical, mechanical and thermal stresses. Therefore, the life of a transformer depends mainly on the condition of the insulation and its ability to withstand the above stresses, during its normal operation. The main reason behind the stresses can be due to aging of insulation and the most critical reason is a short circuit event. As the health of the oil implies the health of the transformer, the oil should be sampled and tested regularly to evaluate the oil condition and to determine the possible fault problems $[1,2]$. Therefore, incipient fault detection of transformers is necessary in order to avoid electrical power system fault as quickly as possible.

The transformer insulation can be classified into mineral oil and solid insulation [3, 4]. Any weakness of insulation may result in failure of the transformer [5]. Insulating oil in a transformer during operation is exposed to a combination of heat, oxygen and electrical discharge, which may lead to its degradation especially through the process of oxidation [6-8]. When an incipient fault occurs in power transformer, either electrical or thermal, causing decomposition of the transformer oil and a number of gases are generated and dissolved into the oil. Such these gases are mainly include Hydrogen $(\mathrm{H} 2)$, methane $(\mathrm{CH} 4)$, Ethane $(\mathrm{C} 2 \mathrm{H} 4)$, Ethylene $(\mathrm{CH} 6)$, acetylene $(\mathrm{C} 2 \mathrm{H} 2)$, carbon monoxide $(\mathrm{CO})$ and carbon dioxide $(\mathrm{CO} 2)$ and the non-fault gases are nitrogen (N2), oxygen (O2). Transformer oil can decompose $\mathrm{CO}$ and $\mathrm{CO} 2$ as a result of its oxidation [8].

Dissolved gas analysis (DGA) is one of the most acceptable techniques used for detecting and evaluating gasses dissolved from mineral oil and also from solid insulation of internal incipient faults in the power transformer based on the oil samples $[9,10]$. It is critically important to monitor the incipient of transformer fault depending on the type and the concentrations of these gases formation. DGA interpretation techniques such as Key gas technique, Doernenbug technique, Roger ratio technique, IEC ratios technique, and Duval 
triangle technique [8], [11-14] are different techniques for the detection of incipient transformer fault conditions.

In this paper, the dissolved gasses were interpreted using several techniques. Different techniques give different analysis result, and it is faced with so much diverse information. These techniques of interpretation of the fault gases are investigated. The study was done to evaluate the accuracy of each technique in predicting the fault based on a fault percentage using MATLAB program.

\section{Samples of Evaluated Transformers}

The evaluation is carried out on DGA data obtained from 352 oil samples has been summarized into 46 samples that have been collected from a 38 different transformers of different rating and different life spans as given in Table 1. It is difficult to determine whether a transformer is behaving normally if it has no previous dissolved gas history. For analyzing the aging degree of the transformer for normal state, we can classify the normal condition as a four status conditions such as: Normal Operation, Caution (investigate), Abnormal (more investigation), and Danger (nearing failure). The DGA guide to classify risks to transformers with no previous problems has been developed by the IEEE [5, 13]. It uses combinations of individual gases and total combustible gas concentration. This guide is not universally accepted and only one of the tools is used to evaluate transformers.

Table 1. Transformer details

\begin{tabular}{|c|c|c|c|c|}
\hline Transf. & No. of transformer & Year of Manufacture & Rating MVA & Voltages KV \\
\hline & & 3- transformer (1981) & 75 & \\
\hline \multirow[t]{2}{*}{ A } & 7 - transformer & 3- transformer (2005) & 125 & $220 / 66 / 11$ \\
\hline & & 1- transformer (2005) & 75 & \\
\hline \multirow[t]{2}{*}{ B } & 2 - transformer & 2- transformer (1982) & 75 & 220/132 \\
\hline & & $\begin{array}{l}\text { 6- transformer }(1990) \\
\text { 2- transformer }(1997) \\
2-\text { transformer }\end{array}$ & & \\
\hline \multirow[t]{4}{*}{ C } & 23 - transformer & 4- transformer (2004) & 25 & $66 / 11$ \\
\hline & & 5- transformer (2005) & & \\
\hline & & 2- transformer (2008) & & \\
\hline & & 2- transformer (2011) & & \\
\hline D & 3 - transformer & $\begin{array}{l}\text { 1- transformer }(1978) \\
\text { 2- transformer }(1980)\end{array}$ & 20 & $66 / 11$ \\
\hline $\mathrm{E}$ & 3 - transformer & $\begin{array}{l}1 \text { - transformer }(1978) \\
2-\text { transformer }(1983)\end{array}$ & 12.5 & $66 / 11$ \\
\hline
\end{tabular}

Table 2 [5], [13] gives one set of guidelines based on good utility practice that is useful for determining the overall health of a power transformer based on the total concentration of combustible gases. The four conditions are defined below:

Table 2. Dissolved key concentration limits in (ppm)

\begin{tabular}{|c|c|c|c|c|}
\hline \multirow{2}{*}{ Gases in (PPM) } & \multicolumn{4}{|c|}{ Gases Status } \\
\hline & Normal & Caution & Abnormal & Danger \\
\hline Hydrogen (PPM) & 100 & $101-700$ & $701-1800$ & $>1800$ \\
\hline Methane (PPM) & 120 & $121-400$ & 401- 1000 & $>1000$ \\
\hline Acetylene (PPM) & 35 & $36-50$ & $51-80$ & $>80$ \\
\hline Ethylene (PPM) & 50 & $51-100$ & $101-200$ & $>200$ \\
\hline Ethane (PPM) & 65 & $66-100$ & $101-150$ & $>150$ \\
\hline Carbon Monoxide PPM) & 350 & $351-570$ & $571-1400$ & $>1400$ \\
\hline Carbon Dioxide (PPM) $^{\mathrm{a}^{\prime}}$ & 2500 & $2501-4000$ & $4001-0000$ & $>10000$ \\
\hline TDCG (PPM) & 720 & $721-1920$ & $1921-4630$ & $>4630$ \\
\hline
\end{tabular}

at is not a combustible gas

\section{Proposed Accurate DGA Technique}

It is worth to mention that in some cases, for each individual traditional interpretation techniques approach such as "key gas, roger's ratio, Doernenburg ratio, IEC ratio and Duval 
triangle" have been given different fault conditions for the same sample unit. To avoid this, the suggested technique gives the appropriate fault based on fault percentage which is given by all the above existing DGA interpretation techniques without any overlapping.

Firstly, check the limit value of the combustible and non combustible gases are applicable or not based on the quantity of each gas concentrations in ppm (parts per million) as an input data for each diagnostic technique as given in table 3quoted from $[5,13]$.

Table 3. Limit concentrations of dissolved gas

\begin{tabular}{lc}
\hline Key gas & Concentrations L1 "ppm" \\
\hline Hydrogen $(\mathrm{H} 2)$ & 100 \\
Methane $(\mathrm{CH} 4)$ & 120 \\
Carbon monoxide $(\mathrm{CO})$ & 350 \\
Acetylene $(\mathrm{C} 2 \mathrm{H} 2)$ & 1 \\
Ethylene $(\mathrm{C} 2 \mathrm{H} 4)$ & 50 \\
Ethane $(\mathrm{C} 2 \mathrm{H} 6)$ & 65 \\
\hline
\end{tabular}

Table 4. Suggested DGA fault types codes for accurate DGA technique

\begin{tabular}{|c|c|c|c|c|c|c|c|}
\hline Technique & $\mathrm{F} 1$ & $\mathrm{~F} 2$ & F3 & $\mathrm{F} 4$ & F5 & F6 & F7 \\
\hline \multirow[t]{3}{*}{ Doernenbug } & Normal & $\begin{array}{l}\text { Out of code } \\
\text { fault }\end{array}$ & \multicolumn{2}{|c|}{ Thermal decomposition } & Arcing & $\begin{array}{c}\text { Partial } \\
\text { Discharges }\end{array}$ & $\begin{array}{l}\text { Out } \\
\text { of } \\
\text { code } \\
\text { fault }\end{array}$ \\
\hline & & $\begin{array}{l}\text { Thermal fault of } \\
\text { low temp }<150\end{array}$ & \multirow[b]{2}{*}{$\begin{array}{c}\text { Thermal fault of } \\
\text { medium temp. } \\
\text { between } 300- \\
700^{\circ} \mathrm{C}\end{array}$} & \multirow[b]{2}{*}{$\begin{array}{c}\text { Thermal } \\
\text { fault of high } \\
\text { temp }>700 \\
{ }^{\circ} \mathrm{C}\end{array}$} & $\begin{array}{l}\text { Discharge } \\
\text { with low } \\
\text { energy }\end{array}$ & & \multirow[b]{2}{*}{$\begin{array}{l}\text { Out } \\
\text { of } \\
\text { code } \\
\text { fault }\end{array}$} \\
\hline & Normal & $\begin{array}{l}\text { Thermal fault of } \\
\text { low temp } \\
\text { between } 150 \text { - } \\
300^{\circ} \mathrm{C}\end{array}$ & & & $\begin{array}{l}\text { Sparking } \\
\text { Discharge } \\
\text { with high } \\
\text { energy, } \\
\text { Arcing }\end{array}$ & $\begin{array}{l}\text { Partial } \\
\text { discharge } \\
\text { with low } \\
\text { energy } \\
\text { density }\end{array}$ & \\
\hline Rogers & Normal & $\begin{array}{c}\text { Thermal fault of } \\
\text { low } \\
\text { temperature } \\
\text { range }<150{ }^{\circ} \mathrm{C} \\
\text { Thermal fault of } \\
\text { temperature } \\
\text { range } 150-200 \\
{ }^{\circ} \mathrm{C} \\
\text { Thermal fault of } \\
\text { temperature } \\
\text { range } 200-300 \\
{ }^{\circ} \mathrm{C}\end{array}$ & $\begin{array}{l}\text { Winding } \\
\text { circulating } \\
\text { current } \\
\\
\text { Core/tank } \\
\text { circulating } \\
\text { current. }\end{array}$ & $\begin{array}{l}\text { Insulated } \\
\text { conductor } \\
\text { overheat }\end{array}$ & $\begin{array}{l}\text { Arc with } \\
\text { power } \\
\text { follows } \\
\text { through. } \\
\text { Sparking. } \\
\text { Flashover. }\end{array}$ & $\begin{array}{c}\text { Partial } \\
\text { discharge } \\
\text { with tracking }\end{array}$ & $\begin{array}{l}\text { Out } \\
\text { of } \\
\text { code } \\
\text { fault }\end{array}$ \\
\hline $\begin{array}{l}\text { Accurate } \\
\text { Technique }\end{array}$ & Normal & $\begin{array}{l}\text { Thermal Fault } \\
\quad<300^{\circ} \mathrm{C}\end{array}$ & $\begin{array}{c}\text { Thermal Fault } \\
300-700^{\circ} \mathrm{C}\end{array}$ & $\begin{array}{c}\text { Thermal } \\
\text { Fault }>700 \\
{ }^{\circ} \mathrm{C}\end{array}$ & Arcing fault & $\begin{array}{c}\text { Partial } \\
\text { discharge }\end{array}$ & $\begin{array}{l}\text { Out } \\
\text { of } \\
\text { code } \\
\text { fault }\end{array}$ \\
\hline
\end{tabular}

Secondly, the same sampling oil unit is analyzed by each computerized individual technique to determine the possible fault types. As an example, for the same sample unit, Key Gas technique got diagnosis condition as "Acing in Oil", Rogers Ratio technique got diagnosis 
condition as "Arc with Power Follow Through", IEC Ratio technique got condition as "No Prediction", and Doernenburg Ratio technique got diagnosis condition as "Arcing" and Duval Triangle technique got diagnosis condition as "Discharge of High Energy". Therefore, for the fault identification with respect to each traditional technique, the faults are categorized into seven types of faults which is assigned with fault codes F1-F7 as suggested in Table 4. The next step is to compare all incipient fault types using each technique. Then, the conclusion is given for final fault type of the tested transformer oil same sample unit based on the percentage given by each techinque. Finally, using MATLAB software all diagnostic results of individual technique and Accurate DGA technique result are displayed.

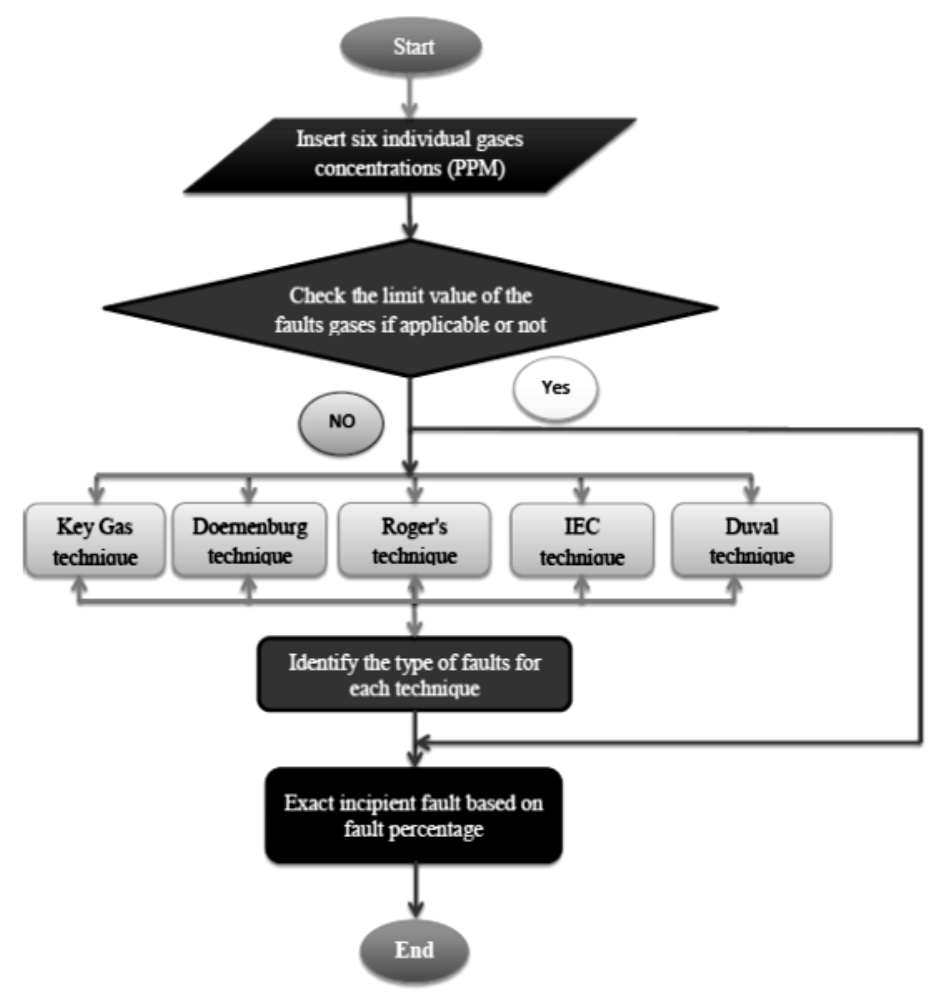

Figure 1. The flowchart of the procedure of Accurate DGA technique

The flowchart on a PC using MATLAB software given in Figure 1 is used in this paper to represent the results of all mentioned techniques. The Accurate DGA technique program will focus on the fault examination analysisidentification using fault percentage analysis to find the accurate fault result, in order to diagnose the incipient faults of the suspected transformers and suggest proper maintenance actions as soon as possible.

\section{Results and Analysis}

Table 5 shows the diagnosis results according to healthy conditions and accurate DGA technique. The developed accurate DGA technique (which is derived from individual traditional techniques) effectively conclude the appropriate fault of the traditional interpretation approach based on fault percentage without any overlapping as it is clear in Table 5 . The suggested technique is based on the fault percentage that is mainlydetermined by using the existing DGA interpretation techniques which are listed in Table 4 without any overlapping, for that resion it is more accurate than any one of them and it is more helpful to detect the transformer faults compared with techniques suggested by Duval, M. [8], Key Gas, IEC ratio, Doernenburg ratioand $\mathrm{R}$. R. Rogers [14]. 
Table 5. Result of fault types codes for individual and accurate DGA technique and transformer state

\begin{tabular}{|c|c|c|c|c|c|c|c|c|c|c|c|c|c|c|}
\hline \multirow{2}{*}{$\begin{array}{l}\mathrm{n} \\
\mathrm{o}\end{array}$} & \multirow[b]{2}{*}{$\mathrm{H}_{2}$} & \multicolumn{6}{|c|}{ Gases in (ppm) } & \multicolumn{5}{|c|}{ Traditional Techniques } & \multirow{2}{*}{$\begin{array}{l}\text { Accur } \\
\text { ate } \\
\text { Techn } \\
\text { ique }\end{array}$} & \multirow{2}{*}{$\begin{array}{l}\text { Transf } \\
\text { ormer } \\
\text { State }\end{array}$} \\
\hline & & $\mathrm{CH}_{4}$ & $\mathrm{C}_{2} \mathrm{H}_{2}$ & $\mathrm{C}_{2} \mathrm{H}_{4}$ & $\mathrm{C}_{2} \mathrm{H}_{6}$ & $\mathrm{CO}$ & $\mathrm{CO} 2^{*}$ & $\begin{array}{c}\text { ey } \\
\text { G } \\
\text { as }\end{array}$ & $\begin{array}{c}\text { Doerne } \\
\text { nburg }\end{array}$ & $\begin{array}{l}\text { Rog } \\
\text { er's }\end{array}$ & $\begin{array}{l}\mathrm{IE} \\
\mathrm{C}\end{array}$ & $\begin{array}{l}\text { Du } \\
\text { val }\end{array}$ & & \\
\hline 1. & $\begin{array}{l}41 \\
\text { Norm } \\
\text { al }\end{array}$ & $\begin{array}{l}65 \\
\text { Norm } \\
\text { al }\end{array}$ & $\begin{array}{l}0 \\
\text { Norm } \\
\text { al }\end{array}$ & $\begin{array}{l}58 \\
\text { Norm } \\
\text { al }\end{array}$ & $\begin{array}{l}22 \\
\text { Norm } \\
\text { al }\end{array}$ & $\begin{array}{l}130 \\
\text { Norm } \\
\text { al }\end{array}$ & $\begin{array}{l}1453 \\
\text { Norm } \\
\text { al }\end{array}$ & $\begin{array}{l}F \\
7\end{array}$ & F1 & F3 & $\begin{array}{l}F \\
3\end{array}$ & F3 & F3 & $\begin{array}{l}\text { Therm } \\
\text { al Fault } \\
300- \\
700^{\circ} \mathrm{C}\end{array}$ \\
\hline 2. & $\begin{array}{l}17 \\
\text { Norm } \\
\text { al }\end{array}$ & $\begin{array}{l}66 \\
\text { Norm } \\
\text { al }\end{array}$ & $\begin{array}{l}0 \\
\text { Norm } \\
\text { al }\end{array}$ & $\begin{array}{l}17 \\
\text { Norm } \\
\text { al }\end{array}$ & $\begin{array}{l}9 \\
\text { Norm } \\
\text { al }\end{array}$ & $\begin{array}{l}268 \\
\text { Norm } \\
\text { al }\end{array}$ & $\begin{array}{l}1362 \\
\text { Norm } \\
\text { al }\end{array}$ & $\begin{array}{l}F \\
7\end{array}$ & F1 & $\mathrm{F} 7$ & $\begin{array}{l}F \\
3\end{array}$ & $\mathrm{~F} 1$ & $\mathrm{~F} 1$ & Normal \\
\hline 3. & $\begin{array}{l}20 \\
\text { Norm } \\
\text { al }\end{array}$ & $\begin{array}{l}48 \\
\text { Norm } \\
\text { al }\end{array}$ & $\begin{array}{l}0 \\
\text { Norm } \\
\text { al }\end{array}$ & $\begin{array}{l}186 \\
\text { Norm } \\
\text { al }\end{array}$ & $\begin{array}{l}36 \\
\text { Norm } \\
\text { al }\end{array}$ & $\begin{array}{l}143 \\
\text { Norm } \\
\text { al }\end{array}$ & $\begin{array}{l}533 \\
\text { Norm } \\
\text { al }\end{array}$ & $\begin{array}{l}F \\
7\end{array}$ & $\mathrm{~F} 1$ & F3 & $\begin{array}{l}F \\
4\end{array}$ & $\mathrm{~F} 4$ & $\mathrm{~F} 4$ & $\begin{array}{l}\text { Therm } \\
\text { al Fault } \\
>700 \\
{ }^{\circ} \mathrm{C}\end{array}$ \\
\hline 5. & $\begin{array}{l}73 \\
\text { Norm } \\
\text { al }\end{array}$ & $\begin{array}{l}177 \\
\text { Cauti } \\
\text { on }\end{array}$ & $\begin{array}{l}0 \\
\text { Norm } \\
\text { al }\end{array}$ & $\begin{array}{l}52 \\
\text { Cauti } \\
\text { on }\end{array}$ & $\begin{array}{l}37 \\
\text { Norm } \\
\text { al }\end{array}$ & $\begin{array}{l}1767 \\
\text { Dang } \\
\text { er }\end{array}$ & $\begin{array}{l}5398 \\
\text { Abno } \\
\text { rmal }\end{array}$ & $\begin{array}{l}F \\
7\end{array}$ & F1 & F3 & $\begin{array}{l}F \\
3\end{array}$ & F3 & F3 & $\begin{array}{l}\text { Therm } \\
\text { al Fault } \\
300- \\
700^{\circ} \mathrm{C}\end{array}$ \\
\hline 6. & $\begin{array}{l}210 \\
\text { Cauti } \\
\text { on }\end{array}$ & $\begin{array}{l}43 \\
\text { Norm } \\
\text { al }\end{array}$ & $\begin{array}{l}187 \\
\text { Dang } \\
\text { er }\end{array}$ & $\begin{array}{l}102 \\
\text { Abno } \\
\text { rmal }\end{array}$ & $\begin{array}{l}12 \\
\text { Norm } \\
\text { al }\end{array}$ & $\begin{array}{l}167 \\
\text { Norm } \\
\text { al }\end{array}$ & $\begin{array}{l}1070 \\
\text { Norm } \\
\text { al }\end{array}$ & $\begin{array}{l}F \\
7\end{array}$ & $\mathrm{~F} 1$ & F7 & $\begin{array}{l}\mathrm{F} \\
5\end{array}$ & F5 & F5 & $\begin{array}{l}\text { Arcing } \\
\text { fault }\end{array}$ \\
\hline 7. & $\begin{array}{l}39 \\
\text { Norm } \\
\text { al }\end{array}$ & $\begin{array}{l}7 \\
\text { Norm } \\
\text { al }\end{array}$ & $\begin{array}{l}0 \\
\text { Norm } \\
\text { al }\end{array}$ & $\begin{array}{l}65 \\
\text { Cauti } \\
\text { on }\end{array}$ & $\begin{array}{l}12 \\
\text { Norm } \\
\text { al }\end{array}$ & $\begin{array}{l}1469 \\
\text { Dang } \\
\text { er }\end{array}$ & $\begin{array}{l}2692 \\
\text { Cauti } \\
\text { on }\end{array}$ & $\begin{array}{l}F \\
4\end{array}$ & $\mathrm{~F} 1$ & $\mathrm{~F} 7$ & $\begin{array}{l}\mathrm{F} \\
7\end{array}$ & $\mathrm{~F} 4$ & $\mathrm{~F} 4$ & $\begin{array}{l}\text { Therm } \\
\text { al Fault } \\
>700 \\
{ }^{\circ} \mathrm{C}\end{array}$ \\
\hline 10. & $\begin{array}{l}18 \\
\text { Norm } \\
\text { al }\end{array}$ & $\begin{array}{l}63 \\
\text { Norm } \\
\text { al }\end{array}$ & $\begin{array}{l}0 \\
\text { Norm } \\
\text { al }\end{array}$ & $\begin{array}{l}51 \\
\text { Cauti } \\
\text { on }\end{array}$ & $\begin{array}{l}20 \\
\text { Norm } \\
\text { al }\end{array}$ & $\begin{array}{l}390 \\
\text { Cauti } \\
\text { on }\end{array}$ & $\begin{array}{l}2633 \\
\text { Cauti } \\
\text { on }\end{array}$ & $\begin{array}{l}F \\
7\end{array}$ & F1 & F7 & $\begin{array}{l}F \\
3\end{array}$ & F3 & F3 & $\begin{array}{l}\text { Therm } \\
\text { al Fault } \\
300^{-} \\
700^{\circ} \mathrm{C}\end{array}$ \\
\hline $\begin{array}{l}11 . \\
12 .\end{array}$ & $\begin{array}{l}200 \\
\text { Cauti } \\
\text { on } \\
28 \\
\text { Norm } \\
\text { al }\end{array}$ & $\begin{array}{l}30 \\
\text { Norm } \\
\text { al } \\
62 \\
\text { Norm } \\
\text { al }\end{array}$ & $\begin{array}{l}98 \\
\text { Dang } \\
\text { er } \\
0 \\
\text { Norm } \\
\text { al }\end{array}$ & $\begin{array}{l}60 \\
\text { Cauti } \\
\text { on } \\
76 \\
\text { Cauti } \\
\text { on }\end{array}$ & $\begin{array}{l}9 \\
\text { Norm } \\
\text { al } \\
16 \\
\text { Norm } \\
\text { al }\end{array}$ & $\begin{array}{l}138 \\
\text { Norm } \\
\text { al } \\
538 \\
\text { Cauti } \\
\text { on }\end{array}$ & $\begin{array}{l}308 \\
\text { Norm } \\
\text { al } \\
1206 \\
\text { Norm } \\
\text { al }\end{array}$ & $\begin{array}{l}F \\
7\end{array}$ & $\mathrm{~F} 1$ & F3 & $\begin{array}{l}\mathrm{F} \\
5\end{array}$ & $\mathrm{~F} 4$ & F5 & $\begin{array}{l}\text { Arcing } \\
\text { fault } \\
\text { Therm } \\
\text { al Fault } \\
>700 \\
{ }^{\circ} \mathrm{C}\end{array}$ \\
\hline 13. & $\begin{array}{l}68 \\
\text { Norm } \\
\text { al }\end{array}$ & $\begin{array}{l}78 \\
\text { Norm } \\
\text { al }\end{array}$ & $\begin{array}{l}0 \\
\text { Norm } \\
\text { al }\end{array}$ & $\begin{array}{l}136 \\
\text { Abno } \\
\text { rmal }\end{array}$ & $\begin{array}{l}103 \\
\text { Abno } \\
\text { rmal }\end{array}$ & $\begin{array}{l}550 \\
\text { Cauti } \\
\text { on }\end{array}$ & $\begin{array}{l}1720 \\
\text { Norm } \\
\text { al }\end{array}$ & $\begin{array}{l}F \\
7\end{array}$ & $F 3, F 4$ & $\mathrm{~F} 7$ & $\begin{array}{l}F \\
3\end{array}$ & $\mathrm{~F} 4$ & $\mathrm{~F} 4$ & $\begin{array}{l}\text { Therm } \\
\text { al Fault } \\
>700 \\
{ }^{\circ} \mathrm{C}\end{array}$ \\
\hline 14. & $\begin{array}{l}54 \\
\text { Norm } \\
\text { al }\end{array}$ & $\begin{array}{l}143 \\
\text { Cauti } \\
\text { on }\end{array}$ & $\begin{array}{l}0 \\
\text { Norm } \\
\text { al }\end{array}$ & $\begin{array}{l}101 \\
\text { Abno } \\
\text { rmal }\end{array}$ & $\begin{array}{l}23 \\
\text { Norm } \\
\text { al }\end{array}$ & $\begin{array}{l}1358 \\
\text { Abno } \\
\text { rmal }\end{array}$ & $\begin{array}{l}4497 \\
\text { Abno } \\
\text { rmal }\end{array}$ & $\begin{array}{l}F \\
7\end{array}$ & $\mathrm{~F} 3, \mathrm{~F} 4$ & F3 & $\begin{array}{l}\mathrm{F} \\
4\end{array}$ & F3 & F3 & $\begin{array}{l}\text { Therm } \\
\text { al Fault } \\
300- \\
700^{\circ} \mathrm{C}\end{array}$ \\
\hline 15. & $\begin{array}{l}19 \\
\text { Norm } \\
\text { al }\end{array}$ & $\begin{array}{l}47 \\
\text { Norm } \\
\text { al }\end{array}$ & $\begin{array}{l}0 \\
\text { Norm } \\
\text { al }\end{array}$ & $\begin{array}{l}62 \\
\text { Cauti } \\
\text { on }\end{array}$ & $\begin{array}{l}27 \\
\text { Norm } \\
\text { al }\end{array}$ & $\begin{array}{l}229 \\
\text { Norm } \\
\text { al }\end{array}$ & $\begin{array}{l}1114 \\
\text { Norm } \\
\text { al }\end{array}$ & $\begin{array}{l}F \\
7\end{array}$ & $\mathrm{~F} 1$ & F3 & $\begin{array}{l}F \\
3\end{array}$ & $\mathrm{~F} 4$ & F3 & $\begin{array}{l}\text { Therm } \\
\text { al Fault } \\
300- \\
700^{\circ} \mathrm{C}\end{array}$ \\
\hline
\end{tabular}











In some cases, Accurate DGA Technique may lead to more than one result (e.g. Accurate Technique in samples number 2, 6, 7, 9, 10, 11, 33, 44). To avoid this in the developed proposed technique, the output will be selected based on the worst case. Consequently, based on MATLAB Code the above analysis is summarized in table 5 . The internal incipient faults diagnosis of power transformer is also accomplished by evaluation of transformer condition using individual and TDCG concentrations for determining the overall health of a power transformer based on four status conditions: Normal Operation, Caution, Abnormal, and Danger (nearing failure). So, the result of this work is useful for planning an appropriate maintenance strategy to keep the power transformer in acceptable conditions. Therefore, incipient fault detection of transformers is necessary in order to avoid electrical power system fault as quickly as possible.

\section{Conclusion}

This paper introduces an accurate DGA technique for identifying the internal incipient faults in the power transformer based on the fault percentage of dissolved gasses released from transformer oil and solid insulation using practical oil samples collected from different transformers. The techniques of interpretation of the fault gases are investigated and compared with each other based on the Accurate DGA technique which is derived from individual techniques to evaluate the accuracy of each technique in predicting the fault based on a MATLAB program as shown in Table 5. Furthermore, the deteriorated transformer by means of this analysis can be carefully focused for the appropriate maintenance as condition-based maintenance before severe damage occurs. 


\section{References}

[1] A Abu-Siada, S Islam. A new approach to identify power transformer criticality and asset management decision based on dissolved gas-in-oil analysis. IEEE Transactions on Dielectrics and Electrical Insulation. 2012; 19: 1007-1012.

[2] Ena Narang, Er Shivanisehgal. Fault Detection Techniques for Maintenance Using Dissolved gas Analysis. International Journal of Engineering Research \& Technology (IJERT). 2012; 1(6): 1-7.

[3] Sherif SM Ghoneim, Sayed A Ward. Dissolved gas Analysis an Early Identification of Transformer Faults. Advances in Electrical Engineering Systems (AEES). 2012; 1(3).

[4] Andri Febriyanto, Tapan Kumar Saha. Oil-immersed Power Transformers Condition Diagnosis with Limited Dissolved Gas Analysis (DGA) Data. Australasian Universities Power Engineering Conference (AUPEC). 2008: 73.

[5] IEEE Guide for the Interpretation of Gases Generated in Oil-Immersed Transformers. C57.104-2008.. Institute of Electrical and Electronics Engineers, Inc. New York: 2008; 9-27.

[6] Siva Sarma, GNS Kalyani. ANN Approach for Condition Monitoring of Power Transformers using DGA. IEEE Region 10 Conference, TENCON 2004. 2004: 444-447.

[7] M Duval. New techniques for dissolved gas-in-oil analysis. IEEE Electrical Insulation Magazine. 2003; 19: 6-15.

[8] Duval M. A Review of Faults Detectable by Gas-in-Oil Analysis in Transformers. IEEE Electrical Insulation Magazine. 2002; 18(3).

[9] DVSS Sivasarma, GNS Kalyani. ANN approach for Condition Monitoring of Power Transformers using DGA. IEEE Electrical Insulation Magazine. 2002: 12-25.

[10] X Liu, F Zhou, F Huang. Research on on-line DGA using FTIR [power transformer insulation testing]. 2002; 3: 1875-1880.

[11] TO Rouse. Mineral insulating oil in transformers. IEEE Electr. Insul. Mag. 1998; 14(3): 6-16.

[12] IEEE and IEC Codes to Interpret Incipient Faults in Transformers, Using Gas in Oil Analysis, by R.R. Rogers C.E.G.B, Transmission Division, Guilford, England. 1995.

[13] IEEE Power Engineering Society. C57.104-1991. IEEE Guide for the Interpretation of Gases Generated in Oil-Immersed Transformers. 1992

[14] RR Rogers. IEEE and IEC Codes to Interpret Incipient Faults in Transformers, Using Gas in Oil Analysis. IEEE Transactions on Electrical Insulation. 1978; 13: 349-354. 